\title{
ON THE DIFFERENTIABILITY OF ISOMETRIES
}

RICHARD S. PALAIS

Let $\mathfrak{T}$ be an $n$-dimensional differentiable manifold of class $C^{k}$, $k=1, \cdots, \infty, \omega$ (as usual $C^{\omega}$ means analytic, and we use the conventions $\infty \pm m=\infty$ and $\omega \pm m=\omega$ for in tegers $m$ ). An automorphism of $\mathfrak{T}$ is a one-to-one map of $\mathfrak{T}$ onto itself which is of class $C^{k}$ and is nonsingular. Now let $\mathfrak{T}$ be given a Riemannian structure, i.e. a positive definite covariant tensor field of rank two, and suppose that in some family of admissible coordinate systems that cover $\mathfrak{T}$ the components of this tensor field are of class $C^{k+1}$. This hypothesis will be satisfied, for example, if $\mathfrak{T}$ is the manifold of class $C^{k}$ associated with a manifold $\tilde{\mathfrak{T}}$ of class $C^{k+2}$ and the metric tensor field is a tensor field of highest possible class (namely $C^{k+1}$ ) on $\tilde{\mathfrak{N}}$. Moreover this hypothesis implies that in the neighborhood of each point of $\mathfrak{T}$ we can define a Riemannian normal coordinate system and that such a coordinate system is admissible, i.e. of class $C^{k}$.

The Riemannian structure on $\mathfrak{T}$ gives rise in a well-known way to a metric $\rho$ on the underlying point set of $\mathfrak{M}$, and we denote by $M$ the metric space that results. An automorphism of $M$ is of course a oneto-one map of $M$ onto itself which preserves distance, i.e. an isometry. A remarkable result of Myers and Steenrod [1] states that an automorphism of $M$ is always an automorphism of $\mathfrak{M}$. It is natural to conjecture that the following stronger result is true.

THEOREM. The differentiable manifold $\mathfrak{T}$ can be canonically reconstructed from the metric space $M$.

We shall show that this is in fact the case. We hope that the construction we give will be illuminating, however it should be admitted in advance that it is not effective in any reasonable sense.

Let $x \in M$. We define a set $M_{x}$ canonically associated with $M$ and called the set of geodesics at $x$ parameterized proportionally to arc length as follows: $\sigma \in M_{x}$ if and only if

(1) $\sigma$ is a map of an interval $(-a, b)$ into $M$ where $a$ and $b$ are positive real numbers or $\infty$.

(2) $\sigma(0)=x$.

(3) There is a real number $r$ such that given

$$
t \epsilon(-a, b) \rho\left(\sigma(t), \sigma\left(t+t^{\prime}\right)\right)=r t^{\prime}
$$

for all sufficiently small $t^{\prime}$.

Received by the editors October 25, 1956. 
(4) $\sigma$ is not a proper restriction of a mapping satisfying the preceding properties.

We next note that if we allow ourselves to use the structure of $\mathfrak{T}$ then we can give $M_{x}$ the structure of an $n$-dimensional real Hilbert space. In fact $\mathfrak{M}_{x}$, the tangent space to $\mathfrak{N}$ at $x$, is a real Hilbert space by virtue of the Riemannian structure for $\mathscr{T}$ and we can carry this structure over to $M_{x}$ by means of the following mapping $\Phi$ : given $v \in \mathfrak{M}_{x}, v \neq 0$ let $\sigma$ be the geodesic with maximal domain which is parameterized by arc length, satisfies $\sigma(0)=x$, and has tangent vector $v /\|v\|$ at 0 , and define $\Phi(v)$ on $(1 /\|v\|)$ (domain $\sigma)$ by $\Phi(v)(t)=\sigma(\|v\| t)$. Of course $\Phi(0)$ is defined as the map $t \rightarrow x$ of $R$ into $M$. The wellknown fact that geodesics parameterized with arc length are just the mappings satisfying (3) above with $r= \pm 1$ insures that $\Phi$ maps $\mathfrak{N}_{x}$ one-to-one onto $M_{x}$. We call the real Hilbert space structure on $M_{x}$ we get in this way its natural real Hilbert space structure. For the moment at least it is not canonically constructed from $M$.

The interesting fact for us is that given the natural real Hilbert space structure on each $M_{x}$ we can reconstruct $\mathscr{T}$. In fact if $\left(e_{1}, \cdots, e_{n}\right)$ is an orthonormal basis for $M_{x}$ then there is a coordinate system $\left(x_{1}, \cdots, x_{n}\right)$ for $\mathfrak{T}$ of class $C^{k}$ defined in a neighborhood $U$ of $x$ by $p=\left(\sum_{i=1}^{n} x_{i}(p) e_{i}\right)(1)$ for $p \in U$. This is of course in the usual terminology just the Riemannian normal coordinate system defined by $\left(\Phi^{-1}\left(e_{1}\right), \cdots, \Phi^{-1}\left(e_{n}\right)\right)$. The above can be stated more elegantly without coordinates as follows: given its natural real Hilbert space structure, $M_{x}$ becomes an $n$-dimensional analytic manifold by requiring that the linear functionals on $M_{x}$ be a base for the analytic structure. With this analytic structure the mapping $\sigma \rightarrow \sigma(1)$ is a nonsingular $C^{k}$ homeomorphism of a neighborhood of 0 in $M_{x}$ onto a neighborhood of $x$ in $\pi$.

Thus to prove our theorem it will suffice to show that the natural real Hilbert space structure of each $M_{x}$ can be constructed canonically from $M$. Now scalar multiplication can be defined even without reference to the metric $\rho$; given $\sigma \in M_{x}$ and a real number $r \neq 0 r \sigma$ is defined by (domain $r \sigma)=(1 / r)$ (domain $\sigma$ ) and $r \sigma(t)=\sigma(r t)$. As for the norm $\|\sigma\|$ of an element $\sigma$ of $M_{x}$, it is just $|r|$ where $r$ is the real number (clearly uniquely determined) occurring in (3) of the defining properties of elements of $M_{x}$. Then since the inner product $\left(\sigma, \sigma^{\prime}\right)$ of two nonzero elements of $M_{x}$ is given by $\|\sigma\| \cdot\left\|\sigma^{\prime}\right\| \cos \theta$ where $\theta$ is the angle between $\sigma /\|\sigma\|$ and $\sigma^{\prime} /\left\|\sigma^{\prime}\right\|$, it follows that we can compute all inner products in $M_{x}$ once we know how to find the cosine of the angle between two elements of norm one. Now an element of $M_{x}$ of norm one is just a geodesic starting from $x$ which is parameterized by 
arc length, and the cosine of the angle $\theta$ between two such elements of $M_{x}, \sigma$ and $\sigma^{\prime}$, can be computed with the aid of the usual trigonometric identities from the formula

$$
\sin \theta / 2=\lim _{s \rightarrow 0}\left(\rho\left(\sigma(s), \sigma^{\prime}(s)\right) / 2 \rho(x, \sigma(s))\right)
$$

which depends only on the metric $\rho$. The validity of the above formula is obvious in the Euclidean case even without the limit, and in the general case it follows from the usual sort of argument that shows that Euclidean formulae hold infinitesimally.

Thus we have shown that we can construct all inner products in $M_{x}$ using only the structure of $M$, and our theorem now follows from the following rather trivial observation.

Lemma. If $H$ is a real Hilbert space then the linear structure of $H$ can be constructed once all inner products are known.

Proof. First we note that an orthonormal base for $H$ can be characterized as a set $\left\{e_{\sigma}\right\}_{\sigma \in \Sigma}$ such that $\left(e_{\sigma}, e_{\sigma^{\prime}}\right)=\delta_{\sigma \sigma^{\prime}}$ for all $\sigma, \sigma^{\prime} \in \Sigma$ and $\left(f, e_{\sigma}\right)=0$ for all $\sigma \in \Sigma$ implies $(f, f)=0$. Choose such a set $\left\{e_{\sigma}\right\}_{\sigma \in \Sigma}$. Given $x$ and $y$ in $H$ and a real number $r, r x$ is characterized in terms of the inner product as the unique element of $H$ such that $\left(r x, e_{\sigma}\right)$ $=r\left(x, e_{\sigma}\right)$ for all $\sigma \in \Sigma$ and similarly $x+y$ is characterized as the unique element of $H$ such that $\left(x+y, e_{\sigma}\right)=\left(x, e_{\sigma}\right)+\left(y, e_{\sigma}\right)$ for all $\sigma \in \Sigma$.

\section{REFERENCES}

1. S. Myers and N. E. Steenrod, The group of isometries of a Riemannian manifold, Ann. of Math. vol. 40 (1939) pp. 400-416.

University of Chicago 